\title{
Impact of electric-field dependent dielectric constants on two-dimensional electron gases in complex oxides
}

\author{
H. Peelaers, ${ }^{1}$ K. Krishnaswamy, ${ }^{2}$ L. Gordon, ${ }^{1}$ D. Steiauf, ${ }^{1}$ A. Sarwe,${ }^{1,3}$ A. Janotti, ${ }^{1, a)}$ \\ and C. G. Van de Walle ${ }^{1}$ \\ ${ }^{1}$ Materials Department, University of California, Santa Barbara, California 93106-5050, USA \\ ${ }^{2}$ Department of Electrical and Computer Engineering, University of California, Santa Barbara, \\ California 93106-9560, USA \\ ${ }^{3}$ Applied Physics Department, Chalmers Institute of Technology, Gothenburg SE 412-96, Sweden
}

(Received 12 August 2015; accepted 24 October 2015; published online 3 November 2015)

\begin{abstract}
High-density two-dimensional electron gas (2DEG) can be formed at complex oxide interfaces such as $\mathrm{SrTiO}_{3} / \mathrm{GdTiO}_{3}$ and $\mathrm{SrTiO}_{3} / \mathrm{LaAlO}_{3}$. The electric field in the vicinity of the interface depends on the dielectric properties of the material as well as on the electron distribution. However, it is known that electric fields can strongly modify the dielectric constant of $\mathrm{SrTiO}_{3}$ as well as other complex oxides. Solving the electrostatic problem thus requires a self-consistent approach in which the dielectric constant varies according to the local magnitude of the field. We have implemented the field dependence of the dielectric constant in a Schrödinger-Poisson solver in order to study its effect on the electron distribution in a 2DEG. Using the $\mathrm{SrTiO}_{3} / \mathrm{GdTiO}_{3}$ interface as an example, we demonstrate that including the field dependence results in the 2DEG being confined closer to the interface compared to assuming a single field-independent value for the dielectric constant. Our conclusions also apply to $\mathrm{SrTiO}_{3} / \mathrm{LaAlO}_{3}$ as well as other similar interfaces. (C) 2015 AIP Publishing LLC. [http://dx.doi.org/10.1063/1.4935222]
\end{abstract}

It is by now well established that a two-dimensional electron gas (2DEG) can form at the interface between two oxides. At $\mathrm{SrTiO}_{3} / \mathrm{GdTiO}_{3}(\mathrm{STO} / \mathrm{GTO})^{1}$ and $\mathrm{SrTiO}_{3} / \mathrm{LaAlO}_{3}$ $(\mathrm{STO} / \mathrm{LAO})^{2}$ interfaces, the high-density electron gas arises from the polar discontinuity ${ }^{2,3}$ present at the interface between a polar and a non-polar material; this provides 0.5 electrons per interface unit cell, ${ }^{4,5}$ corresponding to a maximum sheet carrier density of $3.3 \times 10^{14} \mathrm{~cm}^{-2}$, which has indeed been observed at the STO/GTO interface. ${ }^{1}$ Similar high sheet carrier densities have been observed at $\gamma-\mathrm{Al}_{2} \mathrm{O}_{3} /$ STO interfaces. ${ }^{6}$ Several other oxide interface combinations have been experimentally investigated, ${ }^{7-12}$ and more have been predicted. ${ }^{5}$ Such structures could be used in novel devices analogous to current heterostructure field effect transistors, ${ }^{13-15}$ but with an order of magnitude higher charge density. In addition, the metal-insulator transitions present in some of these complex oxides could enable Mott transistors ${ }^{16}$ with large on/off ratios and femtosecond switching speeds.

The confinement of carriers in an interfacial potential well [inset of Fig. 1] is associated with the presence of a large electric field. The potential profile that confines the carriers is dependent on the dielectric properties of the material. In many complex oxides, including STO, the dielectric properties depend strongly on the electric field, ${ }^{17-19}$ due to the presence of a soft optical phonon mode. ${ }^{20,21}$ Given that the electric field affects the dielectric properties and that the dielectric properties in turn affect the electric field, modeling of the interface based on a Schrödinger-Poisson (S-P) solver should take the field dependence of the dielectric constant

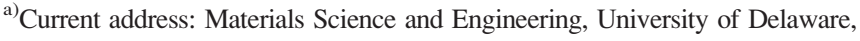
Newark, Delaware 19716-1501, USA
}

into account in a self-consistent manner. Till date, only a few analyses and simulations ${ }^{21-24}$ have accounted for this field dependence at the interface that confines a 2DEG. Taking the field dependence of the dielectric constant into account is not only important in simulations but also necessary for the correct interpretation of experimental $C-V$ measurements at large applied voltages. ${ }^{25}$

In this letter, we perform Schrödinger-Poisson simulations to study the effect of the field-dependent dielectric constant of STO on the 2DEG confined at the interface of STO/ GTO. The simulations are based on parameters obtained from first-principles calculations. While we consider the

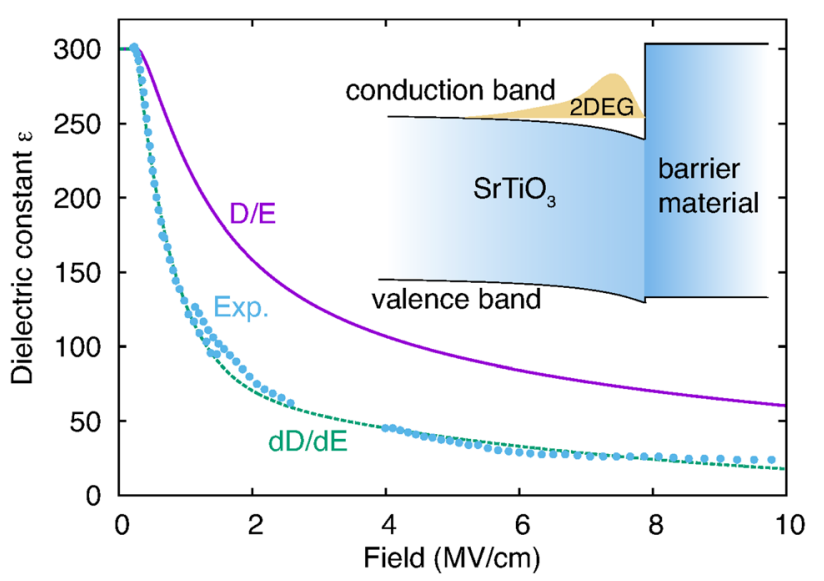

FIG. 1. Dielectric constant as a function of the electric field. The dashed line is determined by fitting Eq. (1) to the experimental measurements of Ref. 19. These experimental data points are not continuous as they were obtained from 3 different samples. The solid line is determined by integrating over the field, as expressed in Eq. (2). The inset shows a schematic band diagram of the confinement of a 2DEG at an interface between STO and a barrier material. The band bending reflects the presence of an electric field. 
STO/GTO heterojunction with the full 2DEG density of $3.3 \times 10^{14} \mathrm{~cm}^{-2}$ as an prototypical example, ${ }^{26}$ the physics described here is more general, and the conclusions are applicable to other heterojunctions with a 2DEG confined in STO, as we will explicitly verify by simulations for a STO/ LAO interface. Our goal is to produce results that can aid in interpreting experiments (e.g., capacitance measurements) and also to provide insight into the physical mechanisms and phenomena that result from the field dependence.

The dependence of the dielectric constant on electric field is taken from experiment ${ }^{19}$ (data points in Fig. 1). In principle, this dependence can also be obtained from firstprinciples calculations, but in practice this requires a large effort that has been executed in only very few instances till date ${ }^{23}$ and then only at $0 \mathrm{~K}$. The dielectric constant is actually strongly temperature dependent, ${ }^{17-19}$ and since the main interest is in devices that operate at room temperature, it is most appropriate to work with the experimental data ${ }^{19}$ that were obtained at room temperature.

Our simulations are based on the nextnano ${ }^{3}$ code $^{27}$ modified to implement a field-dependent dielectric constant. The code solves for the electrostatic potential, charge density, and Fermi level $\left(E_{\mathrm{F}}\right)$ across the heterojunction. The input parameters include electron effective masses $\left(1.0 \mathrm{~m}_{e}\right.$, fitted to reproduce the density of states obtained from density functional theory with a hybrid functional ${ }^{4}$ ) and the static dielectric constant of GTO $(\varepsilon=30)$. The static dielectric constant of STO is implemented as a variable that depends on the electric field. Further details of the implementation are described below. The band gaps of GTO and STO are $2.05 \mathrm{eV}$ and $3.27 \mathrm{eV}$, respectively; the conduction-band offset is $1.47 \mathrm{eV} .^{5}$ The heterojunction was simulated with $500 \mathrm{~nm}$ of STO on one side interfaced with $500 \mathrm{~nm}$ of GTO. No background doping was assumed; we explicitly checked that uniform doping of the STO up to levels of $1 \times 10^{18} \mathrm{~cm}^{-3}$ made no difference to the results (change in $\varepsilon$ smaller than 1 in the region of the highest field). The intrinsic 2DEG of density $3.3 \times 10^{14} \mathrm{~cm}^{-2}$ was simulated by introducing dopants with a concentration of $1.6 \times 10^{22} \mathrm{~cm}^{-3}$ in a $0.2 \mathrm{~nm}$ region on the GTO side of the interface.

Obtaining the field dependence of the dielectric constant from experimental data requires careful consideration of its physical meaning. For linear media, the dielectric constant is the proportionality factor between the electric displacement $D$ and the electric field $E, D=\varepsilon E$. More generally, for non-linear media, $\varepsilon$ depends on the electric field as well, and therefore the previous linear equation should be replaced with a derivative equation: $d D=\varepsilon_{d}(E) d E$, where the subscript in $\varepsilon_{d}(E)$ indicates its derivative nature. We obtained a functional form for the field-dependent dielectric constant by fitting the results of van der Berg et al. ${ }^{19}$ to an empirical equation of the following form:

$$
\varepsilon_{d}(E)= \begin{cases}300, & E \leq 0.27 \mathrm{MV} / \mathrm{cm} \\ \frac{d D}{d E}=a e^{-b E}+c e^{-f E}, & E>0.27 \mathrm{MV} / \mathrm{cm},\end{cases}
$$

where $a=358.50, \quad b=1.84 \mathrm{~cm} / \mathrm{MV}, \quad c=83.84, \quad$ and $f=0.15 \mathrm{~cm} / \mathrm{MV}$ are fitting parameters. At this stage, careful attention should be focused on the form in which the Poisson equation is implemented in the Schrödinger-Poisson solver. In some codes, the Poisson equation is implemented as $E=\int d \mathbf{r}\left[\rho / \varepsilon_{d}(E)\right]$, and $\varepsilon_{d}(E)$ can be used directly. However, other codes (including the nextnano ${ }^{3}$ code we use) implement the Poisson equation as $\nabla \cdot D=\nabla \cdot[\varepsilon(E) E]=\rho$, and therefore, require a dielectric constant that is obtained by integrating Eq. (1) and computing $\varepsilon(E)=\frac{1}{E} \int \frac{d D}{d E} d E$

$$
\varepsilon(E)= \begin{cases}300, & E \leq 0.27 \mathrm{MV} / \mathrm{cm} \\ -\frac{1}{E}\left[\frac{a}{b} e^{-b E}+\frac{c}{f} e^{-f E}-719.307\right], & E>0.27 \mathrm{MV} / \mathrm{cm} .\end{cases}
$$

The experimental data ${ }^{19}$ (three separate curves correspond to three different samples), our fit to the data, and the integrated $\varepsilon(E)$ are all shown in Fig. 1. For electric fields smaller than $0.27 \mathrm{MV} / \mathrm{cm}$, STO behaves as a linear medium; therefore, the dielectric constant remains constant at 300 independent of the field, and the $d D / d E$ and $D / E$ curves coincide.

In practice, the nextnano ${ }^{3}$ code assumes linear media when solving the S-P equation. To mimic a non-linear medium, we partition the STO region of the device into regions with width of $0.1 \mathrm{~nm}$ up to $10 \mathrm{~nm}$ from the interface, and regions with width of $1 \mathrm{~nm}$ between $10 \mathrm{~nm}$ and $50 \mathrm{~nm}$ from the interface. The remaining STO region formed one partition. For the first iteration, all the partitions of STO are assigned a dielectric constant of 300. At the end of each iteration, the electric fields in different regions are obtained from the solution of the S-P solver. Within each region, the average value of the electric field is then used to calculate the corresponding dielectric constant from Eq. (2). The next $\mathrm{S}-\mathrm{P}$ iteration is then performed using the dielectric-constant values assigned to each of the regions, and the electric field is recalculated. We iterate this procedure until the change in the dielectric constant for each region is smaller than 0.01 in consecutive S-P iterations. The converged result is shown in Fig. 2. We checked that the partitioning of the STO into regions was fine enough not to affect the results.

We find that the electric field reaches a maximum of $20.50 \mathrm{MV} / \mathrm{cm}$ at the STO/GTO interface and rapidly decreases away from the interface. In the region of high
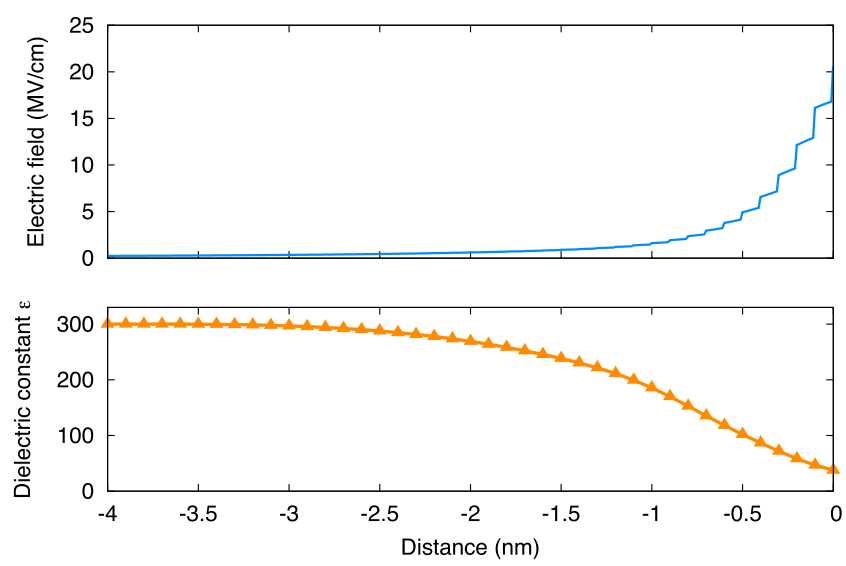

FIG. 2. (a) Calculated electric field near the STO/GTO heterojunction. The junction is located at $0 \mathrm{~nm}$. (b) Corresponding dielectric constant, as determined from Eq. (2) using the calculated average electric field in each region. 


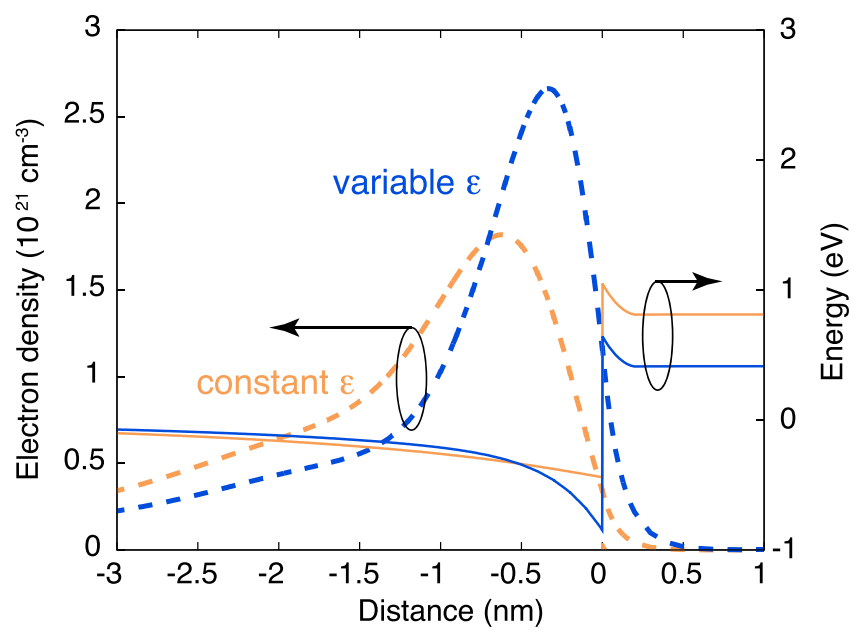

FIG. 3. Electron distribution at the STO/GTO interface (left axis, dashed lines) assuming either a constant dielectric constant $(\varepsilon=300)$ or a fielddependent $\varepsilon$. The corresponding conduction-band profile is shown in solid lines (right axis).

electric field, a corresponding reduction in the dielectric constant is observed [Fig. 2(b)]. Near the interface the dielectric constant in STO is 37 and away from the interface, it increases to the low-field value of 300. Our numerical approach required a discretization of the dielectric constant; due to the large electric fields near the interface and the exponential dependence of $\varepsilon$ on the field, adjacent regions within a few $\AA$ of the interface exhibit large differences in dielectric constant values. This results in discontinuities in the electric field curve near the interface (as $D$ is required to be continuous). Regardless, the values for the electric field and the dielectric constant within each region elucidate the real physical mechanism involved.

The proper treatment of the field dependence of the dielectric constant has a distinct effect on the distribution of electrons within the 2DEG. In Fig. 3, we compare the electron distribution obtained by taking the field dependence into account with the distribution that is obtained using the traditional assumption of a field-independent dielectric constant (with a value of 300). The field dependence reduces the value of the dielectric constant, and from Poisson's equation a smaller dielectric constant results in a larger electric field. Therefore, we expect that taking the field dependence of $\varepsilon$ into account will result in larger electric fields and hence increased band bending as well as stronger confinement of the 2DEG; this is indeed borne out by our simulations, shown in Fig. 3. If a constant $\varepsilon$ is assumed, the 2DEG has a peak density of $1.82 \times 10^{21} \mathrm{~cm}^{-3}$ located $0.62 \mathrm{~nm}$ away from the interface, and $E_{\mathrm{F}}$ lies $0.44 \mathrm{eV}$ above the conduction-band minimum $\left(E_{\mathrm{C}}\right)$ at the interface. The maximum value of the electric field is $2.17 \mathrm{MV} / \mathrm{cm}$, which leads to a reduction of the dielectric constant [Eq. (2)]. Accounting for this field dependence increases $E_{\mathrm{F}}$ to $0.85 \mathrm{eV}$ above $E_{\mathrm{C}}$ and causes the peak in the $2 \mathrm{DEG}$ density to shift closer to the interface by $0.29 \mathrm{~nm}$ compared with the field-independent case. The peak value of the 2 DEG density also increases to $2.66 \times 10^{21} \mathrm{~cm}^{-3}$ (see Table I), and its width is distinctly narrowed compared with the constant- $\varepsilon$ case. Since the screening wavevector in a $2 \mathrm{DEG}$ is inversely proportional to the dielectric constant, ${ }^{28}$ the screening length follows the variation of the dielectric constant [Fig. 2(b)]. The increased electron density also could lead to more electron-electron scattering; ${ }^{29}$ in addition, the closer proximity of the electrons to the interface may lead to increased interfacial scattering.

For systems other than STO/GTO ${ }^{1}$ and $\gamma-\mathrm{Al}_{2} \mathrm{O}_{3} / \mathrm{STO},{ }^{6}$ experimentally reported $2 \mathrm{DEG}$ densities ${ }^{2,13,14}$ are smaller than $3.3 \times 10^{14} \mathrm{~cm}^{-2}$; one possible reason for this reduction is the transfer of electrons from the interface to surface states on the polar material. ${ }^{30}$ It is therefore of interest to address the variation in dielectric constant and band bending for a range of systems with different $2 \mathrm{DEG}$ densities; the results are shown in Table I. We find that 2DEG densities smaller than $1 \times 10^{13} \mathrm{~cm}^{-2}$ leave the dielectric constant unaltered from its low-field value over the entire STO region. In 2DEGs with higher densities, the peak position of the 2DEG shifts towards the interface and its peak value increases. This is accompanied by a larger band bending and hence a larger electric field that decreases $\varepsilon$.

Very similar results were obtained for the STO/LAO interface, in line with existing estimates based on solving the Poisson equation. ${ }^{24}$ This interface differs from the STO/ GTO interface by the conduction-band offset being $1.89 \mathrm{eV}$ instead of $1.47 \mathrm{eV}$. The similarity of the results indicates that the effect of the field dependence is not sensitive to the nature of the wide-band-gap oxide that provides confinement to the 2DEG occurring in STO.

In conclusion, we have studied the effects of the field dependence of the STO dielectric constant on fields and electron distributions in the 2DEG occurring at complex-oxide interfaces. We used Schrödinger-Poisson simulations that self-consistently solve for the electric field and the dielectric constant, with parameters obtained from first-principles calculations along with experimental data for the field dependence. The STO/GTO interface was used as a prototypical interface for our study, but our results apply more generally to other STO interfaces containing a 2DEG such as STO/ LAO, as they are not sensitive to the barrier material confining the 2DEG. The effect of the field dependence is significant, leading to enhanced confinement and increased density of charge carriers closer to the interface. These insights and qualitative results will aid in understanding and analyzing the distribution of charge carriers.

TABLE I. Field-dependent properties of the 2DEG, namely, peak position, peak height, Fermi level relative to conduction-band minimum, minimum value of $\varepsilon$, and maximum value of the electric field, for different $2 \mathrm{DEG}$ densities.

\begin{tabular}{|c|c|c|c|c|c|}
\hline 2DEG density $\left(\mathrm{cm}^{-2}\right)$ & Peak position (nm) & Peak height $\left(10^{20} \mathrm{~cm}^{-3}\right)$ & $\left(E_{\mathrm{F}}-E_{\mathrm{C}}\right)(\mathrm{eV})$ & Minimum $\varepsilon$ & Maximum electric field (MV/cm) \\
\hline $5.0 \times 10^{13}$ & 1.32 & 1.38 & 0.09 & 162 & 3.25 \\
\hline $1.0 \times 10^{14}$ & 1.01 & 3.81 & 0.20 & 104 & 6.48 \\
\hline $3.3 \times 10^{14}$ & 0.33 & 26.6 & 0.85 & 37 & 20.51 \\
\hline
\end{tabular}


We are grateful to A. Alkauskas, S. Rajan, D. Jena, and A. Verma for fruitful discussions. This work was supported by the MURI Program of the Office of Naval Research, Grant No. N00014-12-1-0976 and by the Center for Low Energy Systems Technology (LEAST), one of six SRC STARnet Centers sponsored by MARCO and DARPA. Computational resources were provided by the Center for Scientific Computing at the CNSI and MRL (an NSF MRSEC, DMR-1121053) (NSF CNS-0960316).

${ }^{1}$ P. Moetakef, T. A. Cain, D. G. Ouellette, J. Y. Zhang, D. O. Klenov, A. Janotti, C. G. Van De Walle, S. Rajan, S. J. Allen, and S. Stemmer, Appl. Phys. Lett. 99, 232116 (2011).

${ }^{2}$ A. Ohtomo and H. Y. Hwang, Nature 427, 423 (2004).

${ }^{3}$ J. Lee and A. A. Demkov, Phys. Rev. B 78, 193104 (2008).

${ }^{4}$ A. Janotti, L. Bjaalie, L. Gordon, and C. G. Van De Walle, Phys. Rev. B 86, 241108 (2012).

${ }^{5}$ L. Bjaalie, B. Himmetoglu, L. Weston, A. Janotti, and C. G. Van De Walle, New J. Phys. 16, 025005 (2014).

${ }^{6}$ Y. Z. Chen, N. Bovet, F. Trier, D. V. Christensen, F. M. Qu, N. H. Andersen, T. Kasama, W. Zhang, R. Giraud, J. Dufouleur, T. S. Jespersen, J. R. Sun, A. Smith, J. Nygård, L. Lu, B. Büchner, B. G. Shen, S. Linderoth, and N. Pryds, Nat. Commun. 4, 1371 (2013).

${ }^{7}$ Y. Hotta, T. Susaki, and H. Y. Hwang, Phys. Rev. Lett. 99, 236805 (2007).

${ }^{8}$ H. W. Jang, D. A. Felker, C. W. Bark, Y. Wang, M. K. Niranjan, C. T. Nelson, Y. Zhang, D. Su, C. M. Folkman, S. H. Baek, S. Lee, K. Janicka, Y. Zhu, X. Q. Pan, D. D. Fong, E. Y. Tsymbal, M. S. Rzchowski, and C. B. Eom, Science 331, 886 (2011).

${ }^{9}$ H. Y. Hwang, Y. Iwasa, M. Kawasaki, B. Keimer, N. Nagaosa, and Y. Tokura, Nat. Mater. 11, 103 (2012).

${ }^{10}$ S. Stemmer and S. James Allen, Annu. Rev. Mater. Res. 44, 151 (2014).

${ }^{11}$ Y. Z. Chen, F. Trier, T. Kasama, D. V. Christensen, N. Bovet, Z. I. Balogh, H. Li, K. T. S. Thydén, W. Zhang, S. Yazdi, P. Norby, N. Pryds, and S. Linderoth, Nano Lett. 15, 1849 (2015).

${ }^{12}$ Y. Z. Chen, F. Trier, T. Wijnands, R. J. Green, N. Gauquelin, R. Egoavil, D. V. Christensen, G. Koster, M. Huijben, N. Bovet, S. Macke, F. He, R.
Sutarto, N. H. Andersen, J. A. Sulpizio, M. Honig, G. E. D. K. Prawiroatmodjo, T. S. Jespersen, S. Linderoth, S. Ilani, J. Verbeeck, G. VanTendeloo, G. Rijnders, G. A. Sawatzky, and N. Pryds, Nat. Mater. 14, 801 (2015).

${ }^{13}$ S. Thiel, G. Hammerl, A. Schmehl, C. W. Schneider, and J. Mannhart, Science 313, 1942 (2006).

${ }^{14}$ C. Cen, S. Thiel, J. Mannhart, and J. Levy, Science 323, 1026 (2009).

${ }^{15}$ B. Förg, C. Richter, and J. Mannhart, Appl. Phys. Lett. 100, 053506 (2012).

${ }^{16}$ J. Son, S. Rajan, S. Stemmer, and S. James Allen, J. Appl. Phys. 110, 084503 (2011).

${ }^{17}$ H.-M. Christen, J. Mannhart, E. J. Williams, and C. Gerber, Phys. Rev. B 49, 12095 (1994).

${ }^{18}$ J. Hemberger, P. Lunkenheimer, R. Viana, R. Böhmer, and A. Loidl, Phys. Rev. B 52, 13159 (1995).

${ }^{19}$ R. A. van der Berg, P. W. M. Blom, J. F. M. Cillessen, and R. M. Wolf, Appl. Phys. Lett. 66, 697 (1995).

${ }^{20}$ R. A. Cowley, Phys. Rev. A 134, A981 (1964).

${ }^{21}$ G. Khalsa and A. MacDonald, Phys. Rev. B 86, 125121 (2012).

${ }^{22}$ K. V. Reich, M. Schecter, and B. I. Shklovskii, Phys. Rev. B 91, 115303 (2015).

${ }^{23}$ M. Stengel, Phys. Rev. Lett. 106, 136803 (2011).

${ }^{24}$ O. Copie, V. Garcia, C. Bödefeld, C. Carrétéro, M. Bibes, G. Herranz, E. Jacquet, J.-L. Maurice, B. Vinter, S. Fusil, K. Bouzehouane, H. Jaffrès, and A. Barthélémy, Phys. Rev. Lett. 102, 216804 (2009).

${ }^{25}$ D. Kahng and S. H. Wemple, J. Appl. Phys. 36, 2925 (1965).

${ }^{26}$ The band diagram for STO/GTO differs from the one shown in the inset of Fig. 1 by the inclusion of a "lower Hubbard band" in the GTO, lying well below the conduction-band minimum of STO (see Ref. 5). The presence of this band does not affect any of the physics discussed in the present work.

${ }^{27}$ S. Birner, S. Hackenbuchner, M. Sabathil, G. Zandler, J. A. Majewski, T. Andlauer, T. Zibold, R. Morschl, A. Trellakis, and P. Vogl, Acta Phys. Pol. A 110, 111 (2006).

${ }^{28}$ T. Ando, A. B. Fowler, and F. Stern, Rev. Mod. Phys. 54, 437 (1982).

${ }^{29}$ E. Mikheev, B. Himmetoglu, A. P. Kajdos, P. Moetakef, T. A. Cain, C. G. Van de Walle, and S. Stemmer, Appl. Phys. Lett. 106, 062102 (2015).

${ }^{30}$ K. Krishnaswamy, C. E. Dreyer, A. Janotti, and C. G. Van de Walle, Phys. Rev. B 92, 085420 (2015) 